\title{
Saturated patterned excitation microscopy-a concept for optical resolution improvement
}

\author{
Rainer Heintzmann and Thomas M. Jovin \\ Max-Planck Institute for Biophysical Chemistry, Am Fassberg 11, 37077 Göttingen, Germany \\ Christoph Cremer \\ Kirchhoff-Institute of Physics, University of Heidelberg, Albert-Ueberle-Strasse 3-5, 69120 Heidelberg, Germany
}

Received July 31, 2001; revised manuscript received January 14, 2002; accepted March 7, 2002

\begin{abstract}
The resolution of optical microscopy is limited by the numerical aperture and the wavelength of light. Many strategies for improving resolution such as $4 \mathrm{Pi}$ and $\mathrm{I}^{5} \mathrm{M}$ have focused on an increase of the numerical aperture. Other approaches have based resolution improvement in fluorescence microscopy on the establishment of a nonlinear relationship between local excitation light intensity in the sample and in the emitted light. However, despite their innovative character, current techniques such as stimulated emission depletion (STED) and ground-state depletion (GSD) microscopy require complex optical configurations and instrumentation to narrow the point-spread function. We develop the theory of nonlinear patterned excitation microscopy for achieving a substantial improvement in resolution by deliberate saturation of the fluorophore excited state. The postacquisition manipulation of the acquired data is computationally more complex than in STED or GSD, but the experimental requirements are simple. Simulations comparing saturated patterned excitation microscopy with linear patterned excitation microscopy (also referred to in the literature as structured illumination or harmonic excitation light microscopy) and ordinary widefield microscopy are presented and discussed. The effects of photon noise are included in the simulations. () 2002 Optical Society of America
\end{abstract}

OCIS codes: $180.2520,110.2990,110.0180,110.4850,190.1900,170.0180$.

\section{INTRODUCTION}

The resolution of optical systems is generally limited by the numerical aperture (NA) of the objective lens system and the wavelength of the light. The fundamental resolution limit of a light microscope is given by the extent of the nonvanishing part of its optical transfer function (OTF), which is the Fourier transform of the point-spread function (PSF). This region of support defines the extent to which spatial frequencies composing the object are either conserved during imaging or attenuated and possibly phase shifted. Without further assumptions about the object (as, for example, signal positivity or a specified, limited spatial extent), information about the spatial frequencies lost in the imaging process cannot be recovered. Accordingly, expanding the region of support of the OTF has been a major goal of strategies for achieving superresolution in microscopy. ${ }^{1-8}$

In fluorescence microscopy one generally aims for conditions ensuring that the emitted fluorescence is proportional to the local intensity (or, more specifically, the irradiance) of the illumination light; i.e., the saturation limit is avoided by use of low irradiance. In contrast to transmitted or reflected light, fluorescence is incoherent owing to its large spectral width and stochastic nature (dephasing). The measured image $I(\mathbf{x})$ (transformed back into object space coordinates $\mathbf{x}$ ) can thus be described by a multiplication of the local excitation intensity $I_{\mathrm{ex}}(\mathbf{x})$ by the local fluorophore concentration $\rho(\mathbf{x})$, followed by a convolution (denoted by $\otimes$ ) with the PSF $h_{\mathrm{em}}(\mathbf{x})$ of the incoherent imaging system for the emitted light:

$$
I(\mathbf{x})=h_{\mathrm{em}}(\mathbf{x}) \otimes\left(I_{\mathrm{ex}}(\mathbf{x}) \rho(\mathbf{x})\right) .
$$

For simplicity, constant factors will henceforth be omitted if they are not important in defining the final structure of the image. In reciprocal space, Eq. (1) translates into a convolution of the Fourier-transformed excitation intensity distribution $\widetilde{I_{\text {ex }}}(\mathbf{k})$ with the Fourier transformed object density distribution $\widetilde{\rho}(\mathbf{k})$ followed by multiplication with the OTF $\overparen{h_{\mathrm{em}}}(\mathbf{k})$. The tilde above a function denotes a Fourier transformation, and $\mathbf{k}$ represents the spatialfrequency vector in reciprocal space:

$$
\widetilde{I}(\mathbf{k})=\widetilde{h_{\mathrm{em}}}(\mathbf{k})\left(\widetilde{I_{\mathrm{ex}}}(\mathbf{k}) \otimes \widetilde{\rho}(\mathbf{k})\right) .
$$

In general, many incoherent microscopy techniques can be described by using Eqs. (1) and (2) and interpreting $\rho(\mathbf{x})$ as the spatial density distribution of a particular property of the sample. In the case of iterative nonlinear reconstruction techniques, this consideration does not apply exactly but often holds to a reasonable approximation.

The limitations imposed by the NA and the wavelength of light restrict the region of support to a finite domain. This principle applies to the detection OTF and similarly to the Fourier transform of the illumination distribution $\widetilde{I_{\text {ex }}}(\mathbf{k})$ of fluorescence excitation. Many approaches have sought to maximize the extent of both of these functions for resolution improvement ("PSF engineering"). The principal improvements over standard widefield microscopy in conformance with Eq. (1) are confocal microscopy, ${ }^{9}$ standing wave-field microscopy, ${ }^{10-12} 4 \mathrm{Pi}^{-8,13,14}$ and $I^{5} M-,{ }^{15-17}$ aperture correlation microscopy, ${ }^{18,19}$ patterned 
excitation $^{20}$ microscopy, ${ }^{1-7}$ and axial tomography. ${ }^{21-23}$ However, all of these methods are still fundamentally limited by the Abbé limit defining the region of transferable spatial frequencies. It is therefore desirable to enlarge this region of transferable frequencies by using a different strategy, one of which is to break the linear relationship expressed by Eq. (1).

A nonlinear relationship is exploited in two-photon or multiphoton microscopy. ${ }^{24-26}$ However, in this case the nonlinearity is achieved at the price of a longer excitation wavelength, such that the improvement in resolution is only modest. Recent techniques based on nonlinearities realized or proposed by Hell and co-workers are groundstate depletion (GSD) microscopy pumping the triplet state, ${ }^{27,28}$ stimulated-emission depletion microscopy (STED), ${ }^{29,30}$ and strategies based on fluorescence resonance energy transfer ${ }^{31}$ or prolongation of the excitedstate lifetime by repetitive excitation. ${ }^{32}$ STED and GSD rely primarily on a saturation mechanism (involving either the stimulated emission and/or the saturation of the triplet state) to achieve the desired nonlinearity. However, all of these methods require complex experimental designs to achieve the goal of resolution improvement beyond the Abbe limit. The new method described here increases in the transmittable spatial frequencies without the need for stimulated emission or complicated depletion schemes.

\section{THEORY OF NONLINEAR RESOLUTION IMPROVEMENT}

We utilize the fact that under nonlinear conditions, Eq. (1) can be generalized to

$$
\begin{aligned}
& h_{\mathrm{em}}(\mathbf{x}) \otimes I_{\mathrm{em}}\left(\rho(\mathbf{x}), I_{\mathrm{ex}}(\mathbf{x})\right), \\
& \Leftrightarrow \widetilde{h_{\mathrm{em}}}(\mathbf{k}) \widetilde{I_{\mathrm{em}}}\left(\rho(\mathbf{k}), I_{\mathrm{ex}}(\mathbf{k})\right),
\end{aligned}
$$

in which the linear relationship between the spatial distribution of the illumination intensity $I_{\mathrm{ex}}(\mathbf{x})$ and the emitted light intensity $I_{\mathrm{em}}(\mathbf{x})$ has been dropped. Instead, $I_{\mathrm{em}}(\mathbf{x})$ is now given as a function depending on the local fluorophore concentration $\rho(\mathbf{x})$ and $I_{\mathrm{ex}}(\mathbf{x})$. Assuming a linear dependence of $I_{\mathrm{em}}(\mathbf{x})$ on $\rho(\mathbf{x})$ (as in fluorescence microscopy), the emitted intensity $I_{\mathrm{em}}(\mathbf{x})$ can be approximated by a Taylor series expansion with constant coefficients $c_{i}$ :

$$
\begin{aligned}
I_{\mathrm{em}}(\mathbf{x}) \cong & c_{0}+c_{1} \rho(\mathbf{x})+c_{2} I_{\mathrm{ex}}(\mathbf{x})+c_{3} \rho(\mathbf{x}) I_{\mathrm{ex}}(\mathbf{x}) \\
& +c_{4} \rho(\mathbf{x}) I_{\mathrm{ex}}(\mathbf{x})^{2}+c_{5} \rho(\mathbf{x}) I_{\mathrm{ex}}(\mathbf{x})^{3}+\cdots
\end{aligned}
$$

The term connected to $c_{3}$ corresponds to the linear case stated in Eq. (1). Neglecting constant offsets, relation (5) can be further simplified to

$$
\begin{aligned}
I_{\mathrm{em}}(\mathbf{x}) \cong & \rho(\mathbf{x})\left[c_{1}+c_{3} I_{\mathrm{ex}}(\mathbf{x})+c_{4} I_{\mathrm{ex}}(\mathbf{x})^{2}\right. \\
& \left.+c_{5} I_{\mathrm{ex}}(\mathbf{x})^{3}+\cdots\right], \\
I_{\mathrm{em}}(\mathbf{x})= & \rho(\mathbf{x}) E m(\mathbf{x}),
\end{aligned}
$$

where

$$
\operatorname{Em}(\mathbf{x})=\sum_{i=1}^{\infty} c_{i} I_{\mathrm{ex}}(\mathbf{x})^{i} .
$$

The newly introduced proportionality term will be denoted the spatially dependent emittability $\operatorname{Em}(\mathbf{x})$ of the object. In the linear case [Eq. (1)], $\operatorname{Em}(\mathbf{x})=c_{3} I_{\mathrm{ex}}(\mathbf{x})$.

The Fourier-transformed emission intensity $\widetilde{I_{\mathrm{em}}}\left(\rho(\mathbf{k}), I_{\mathrm{ex}}(\mathbf{k})\right)$ is now given by

$$
\widetilde{I_{\mathrm{em}}}(\mathbf{k})=\widetilde{\rho}(\mathbf{k}) \otimes \widetilde{E m}(\mathbf{k})
$$

In analogy to Eq. (2), Eq. (8) contains the term $c_{3} \widetilde{I_{\mathrm{ex}}}(\mathbf{k})$ $\otimes \widetilde{\rho}(\mathbf{k})$, since Fourier transformation is a linear operation. Assuming a spatially periodic $I_{\mathrm{ex}}(\mathbf{x}), \widetilde{I_{\mathrm{ex}}}(\mathbf{k})$ can be decomposed into a finite sum of $\delta$ distributions. Thus, depending on the distribution of the incident light intensity, the components of $\widetilde{\rho}(\mathbf{k})$ will be shifted in Fourier space and summed with varying weights [Fig. 1(b)], a process described by $\widetilde{I_{\text {ex }}}(\mathbf{k}) \otimes \widetilde{\rho}(\mathbf{k})$ in Eq. (2).

The optical imaging of the emitted intensity distribution $I_{\mathrm{em}}(\mathbf{x})$ causes only a certain region in Fourier space, given by the region of support of the OTF $\overparen{h_{\mathrm{em}}}(\mathbf{k})$, to be transferred. Information at spatial frequencies outside this region is suppressed completely. The region of detectable object spatial frequencies with use of patterned excitation, however, is enlarged owing to the illumination with a spatially dependent intensity distribution [Fig. 1(b)], which shifts object spatial frequencies into the region of support of the detection OTF. With appropriate image-reconstruction algorithms, the shifted object spatial frequencies can be repositioned so as to achieve a consistent, high-resolution image. Such a reconstruction based on a one-dimensional, linear model has been described. ${ }^{1-7}$ These methods enlarge the region of support of the OTF by a factor of $\sim 2$, i.e., that which can be achieved by confocal fluorescence microscopy. However, in comparison with confocal techniques, patterned excitation methods are more sensitive to the detection of high spatial frequencies.

When nonlinearities are present [relation (6)], higherorder terms in $I_{\text {ex }}(\mathbf{x})$ will be finite in $\operatorname{Em}(\mathbf{x})$ [Eq. (7)], leading to corresponding terms $c_{4} \quad\left[\widetilde{I_{\text {ex }}}(\mathbf{k}) \otimes \widetilde{I_{\text {ex }}}(\mathbf{k})\right]$ $\otimes \widetilde{\rho}(\mathbf{k})$ in the Fourier transform, $\widetilde{E m}(\mathbf{k})$ [Eq. (8)]. The positions of the resulting peaks of the emittability pattern now lie beyond the limiting spatial frequency given by the excitation OTF, which is evident from inspecting the emittability structure generated by the term $\widetilde{I_{\mathrm{ex}}}(\mathbf{k}) \otimes \widetilde{I_{\mathrm{ex}}}(\mathbf{k})$. Although $\widetilde{h_{\mathrm{em}}}(\mathbf{k})$ permits only the detection of rather low spatial frequencies of the emission pattern, the attachment of the Fourier-transformed object to these highfrequency peaks [Fig. 1(d)] permits the detection of object spatial frequencies beyond the limits of linear patterned excitation [Eq. (2), Fig. 1(b)]. That is, in the nonlinear case higher-spatial-frequency information is shifted into the range of spatial frequencies detectable by the $\widetilde{h_{\mathrm{em}}}(\mathbf{k})$, to a degree determined by the order of the respective nonlinear term in $\overparen{E m}(\mathbf{k})$. In theory, this circumstance permits the detection of object information at arbitrarily high spatial frequencies and therefore with arbitrary resolution, although in practice the achievable resolution will be limited by the signal-to-noise ratio (SNR) of the raw data. 


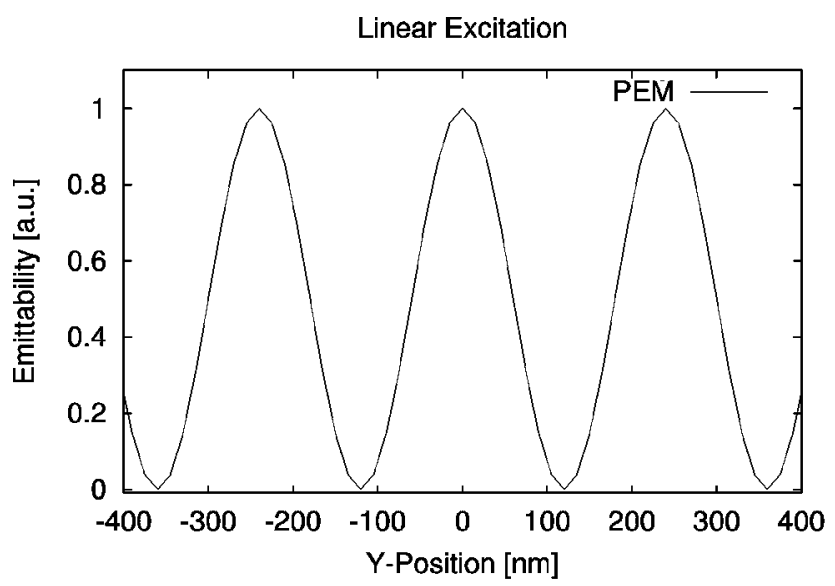

(a)

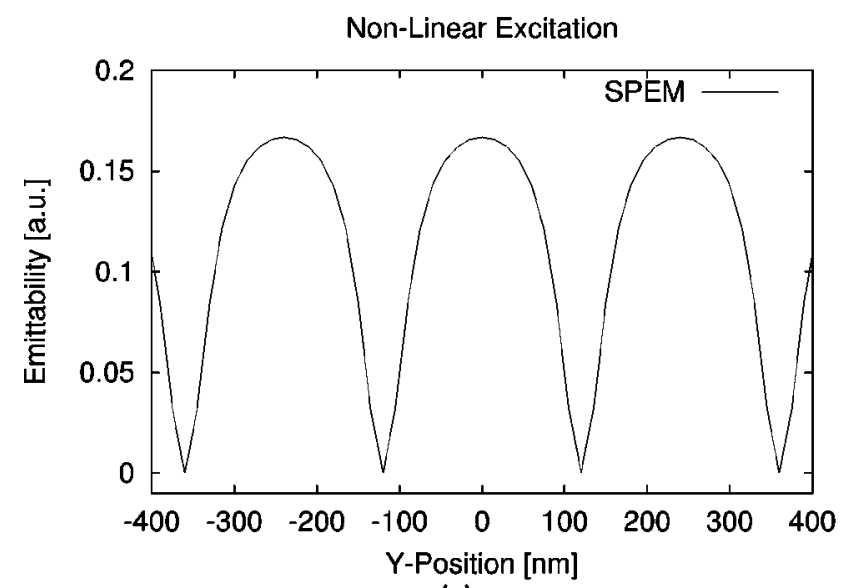

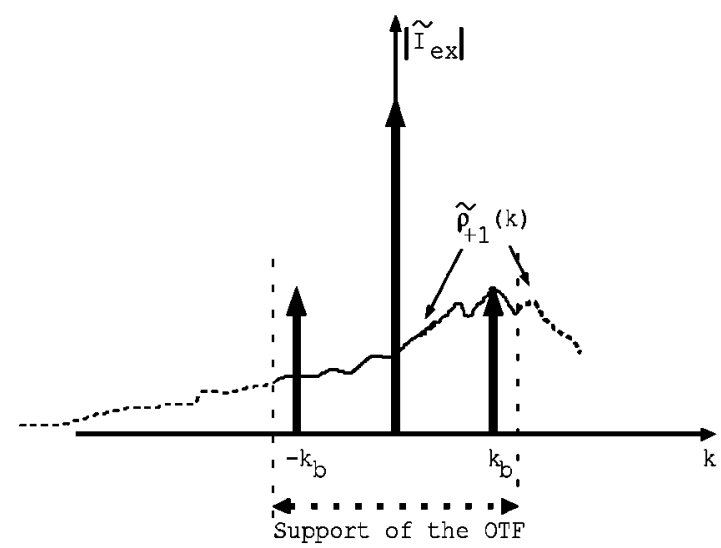

(b)

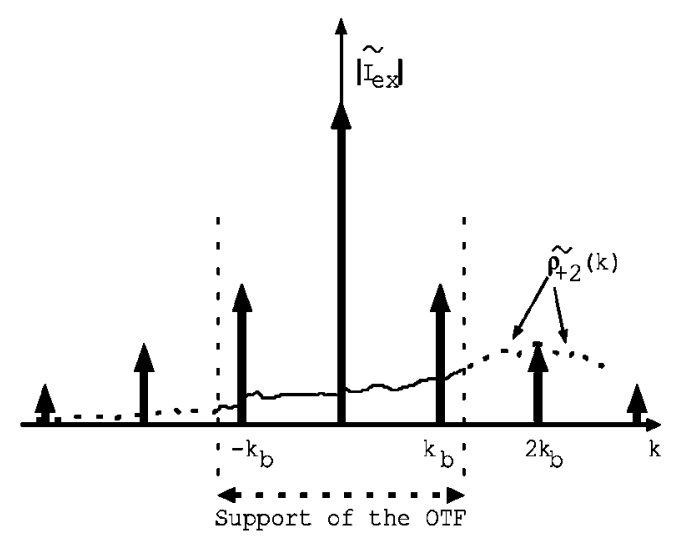

(d)

Fig. 1. Fundamental concept of SPEM implemented by fluorophore saturation. (a) Pattern of the sinusoidal emittability distribution generated by low-intensity patterned excitation (linear patterned excitation microscopy). (b) Scheme of the emittability pattern of (a) in Fourier space. The vertical arrows denote the frequency maxima corresponding to the sinusoidal pattern of the excitation. The structure of the Fourier-transformed object density distribution $\widetilde{\rho_{+1}}(k)$ attached to one of those maxima is also shown. Other such components are omitted for clarity. (c) Emittability distribution in real space with high-intensity illumination leading to fluorophore saturation. This emittability distribution was used in the SPEM simulations. (d) Corresponding emittability pattern in Fourier space. The arrows denote the maxima, which are caused by the nonlinear distortion (by fluorophore saturation) of the sinusoidal intensity pattern (a), (b). The Fourier-transformed object distribution is attached to every maximum [for clarity this is shown only for the second maximum $\left.\widetilde{\rho_{+2}}(k)\right]$.

\section{SATURATION AS NONLINEAR PROCESS}

A possible realization of the concept embodied in Eq. (8) is to use fluorescence saturation as a nonlinear process relating emission to excitation. In a two-state system (i.e., for the moment omitting the triplet state) the emission from the first excited singlet state ${ }^{33,34}$ is given by

$$
I_{\mathrm{em}} \propto \frac{k_{f} \Psi_{\mathrm{ex}}}{\frac{1}{\sigma \tau}+\Psi_{\mathrm{ex}}},
$$

where $k_{f}$ is the radiation rate constant, $\sigma$ the absorption cross section, and $\tau$ the fluorescence lifetime of the fluorophore; $\Psi_{\mathrm{ex}}$ is the photon flux proportional to $I_{\mathrm{ex}}$. In the limiting case of a very small $\sigma \tau$ or low $\Psi_{\text {ex }}$, relation (9) is reduced to a linear dependence of $I_{\mathrm{em}}$ on $\Psi_{\mathrm{ex}}$. In the case of a large $\sigma \tau$ or a high $\Psi_{\text {ex }}, I_{\text {em }}$ reaches a plateau $\propto k_{f}$. The presence of a triplet state will alter the saturation behavior slightly, leading to a plateau at a lower emission intensity. However, the triplet state, if it exists, does not change the essential characteristics of fluorescence saturation. In fact, triplet-state saturation is the basis of GSD and could have practical advantages in SPEM.

Saturation can be achieved by illumination with a high-power sinusoidal spatial intensity distribution. As described above, the detected light will contain spatial frequencies of the object distribution otherwise inaccessible in the linear regime. However, the mixed contributions representing the high object spatial-frequency information in the raw image need to be processed appropriately to achieve the desired high-resolution image.

As described in Appendix A, a system of equations can be derived and solved at every pixel position, assuming a spatial shift of the intensity $I_{\mathrm{ex}}(\mathbf{x})$ and thus the emittability pattern $\operatorname{Em}(\mathbf{x})$. Similar approaches have been used for the linear case. ${ }^{1-7}$ It should be stressed that fluorescence saturation is an attractive, but by no means unique, mechanism for obtaining the required nonlinearity. 


\section{VIRTUAL MICROSCOPY SIMULATIONS}

Figure 2 shows possible realizations of the SPEM concept by far-field epi-fluorescence microscopy. Figure 3 displays a series of simulated SPEM images, based on the arrangement given in Fig. 2(a), with a one-dimensional sinusoidal grating as a spatial light modulator (SLM) and blockage of the zero diffraction order (see also Section 5). The intensity is displayed as gray levels. A constant background fluorescence was added to the object for better visualization of the emittability pattern and to simulate possible background fluorescence [Fig. 3(a)]. To simulate a worst-case scenario, the background level was relatively high, corresponding to $\sim 7000$ photons (out of a maximum of $10^{4}$ photons in the simulated images). The grating period [Fig. 2(a)] was chosen such that only the 0 , the +1 , and the $-1 \delta$ peaks in Fourier space were represented in the illumination intensity distribution (as would be obtained in practice by using two diffraction orders, +1 and -1 , for illumination). In simulations with saturation (SPEM), $m= \pm 3$ orders were accounted for in each of the four illumination directions (horizontal, vertical, and the two diagonals). The first orders were placed at $\sim 83 \%$ (horizontal and vertical) and $88 \%$ (for both diagonal directions) of the in-plane border frequency of the

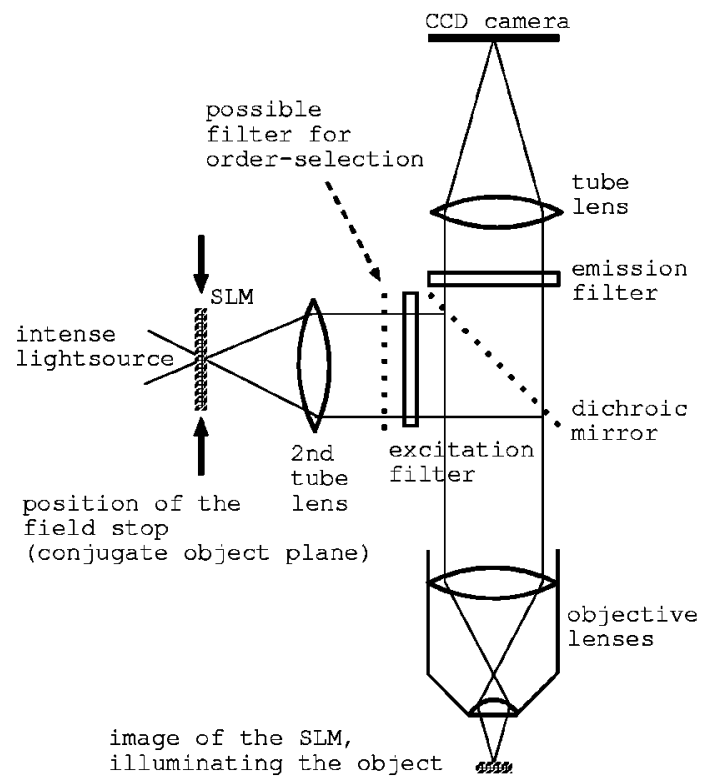

(a)

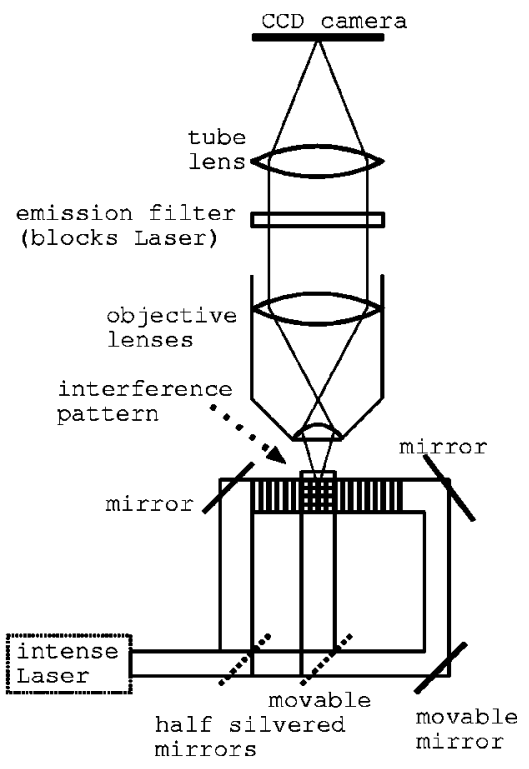

(b)

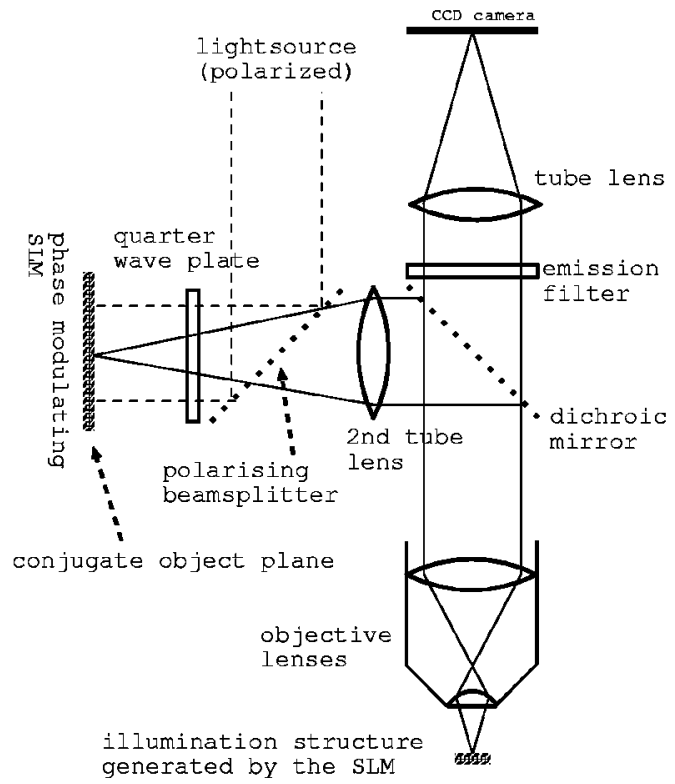

(c)

Fig. 2. Possible realizations of the SPEM concept by far-field epi-fluorescence microscopy. (a) The SLM could be a diffraction pattern generated by a mask, an LCD, or a digital mirror device. To introduce the nonlinearity, a very bright light source is needed. (b) Implementation with a coherent laser source for the generation of standing waves. (c) Improved version of (a) with use of a phase-modulating SLM to permit a high degree of modulation as well as a high light efficiency on the illumination side. 


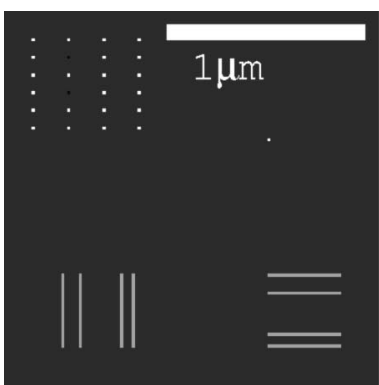

(a)

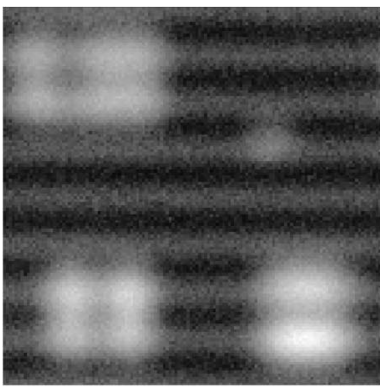

(c)

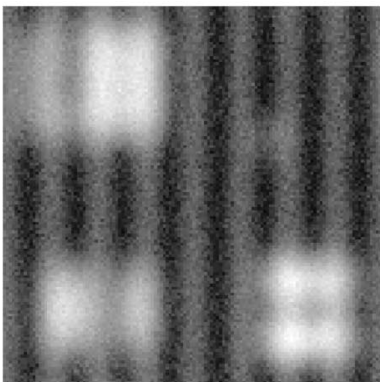

(e)

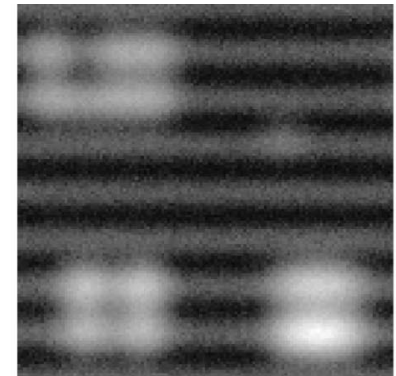

(b)

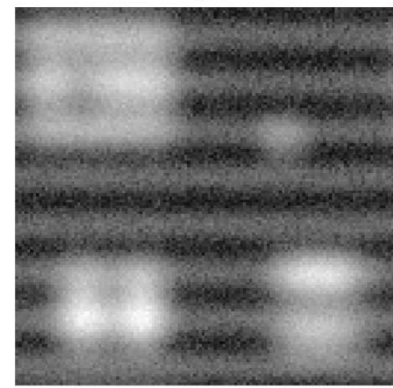

(d)

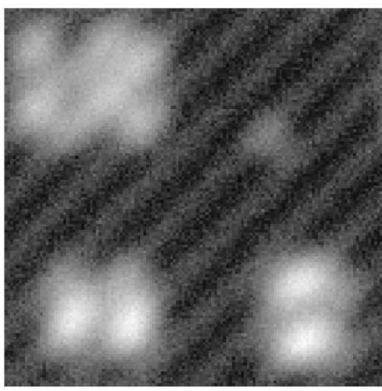

(f)
Fig. 3. Simulations of resolution enhancement in SPEM. (a) The object, displayed with a slight clipping at high intensities. The object was simulated to contain a background fluorescence level (resulting in $\sim 7000$ expected photons per pixel in its image) so that the grating pattern can be observed in the images. (b)(f) Virtual microscopic images simulated with a theoretic PSF and added Poisson noise (maximum pixel set to $10^{4}$ expected photons). (b) Simulated image under linear patterned excitation conditions (phase 1). (c), (d) Images simulated at phase 1 and phase 4 of the illumination pattern (out of 7 phases) with a relative saturation of $\alpha=5 / 6$. (e), (f) Other images simulated at different directions of patterning.

widefield fluorescence emission OTF (which should be simple to achieve practically, since the excitation wavelength is shorter than the emission wavelength). The widefield OTF was simulated at a $520 \mathrm{~nm}$ emission wavelength with vector theory $(\mathrm{NA}=1.3, n=1.518)$ with aplanar apodization. ${ }^{35}$ All simulations were performed on a two-dimensional $(128 \times 128)$ grid with a $15-\mathrm{nm}$ pixel spacing. The reconstructed result $I_{\text {rec }}$ was Fourier filtered by using the magnitude of the fast Fourier transform of a reconstructed noise-free simulated single point object $P_{\text {rec }}$ and a regularization parameter $\gamma$ to yield the high-frequency enhanced result:

$$
\widetilde{I_{\mathrm{hfe}}}=\widetilde{I_{\text {rec }}} \frac{1}{\mid \widetilde{P_{\text {rec }} \mid}+\gamma} .
$$

The regularization parameter $\gamma$ was selected to be $2 \%$ of the maximum of $\left|\widetilde{P_{\text {rec }}}\right|$ (which occurs at zero spatial fre- quency). A different method of filtering might conceivably improve the results further.

To simulate saturation, a model based on relation (9) was chosen, from which the fraction $\alpha$ of the maximum possible emission (the relative saturation) can be deduced:

$$
\alpha=\frac{\Psi_{\mathrm{ex}}}{\frac{1}{\sigma \tau}+\Psi_{\mathrm{ex}}} .
$$

In the data simulating saturation that are presented below, $\alpha$ was selected to be $5 / 6$ at the point of maximum excitation intensity in each image.

In Fig. 4, reconstructed images are compared with conventional far-field fluorescence imaging coupled to a highfrequency filtering technique [Eq. (10)] and with a linear patterned excitation technique. It is apparent that the SPEM method greatly enhances image quality. In Figs. 5 and 6 the PSFs and OTFs of the different methods are compared. In Fig. 5(f) the greatly extended region of support of the OTF in comparison with the linear case [Fig. 5(e)] is observed. It is apparent that for the number of orders $(m= \pm 3)$ accounted for, more patterning direc-

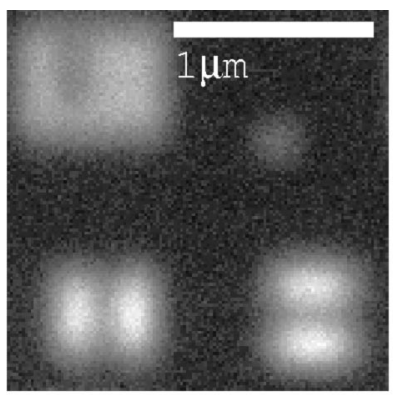

(a)

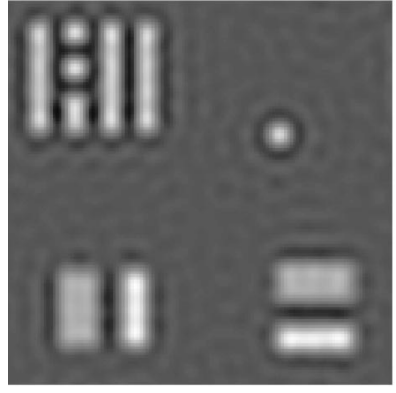

(c)

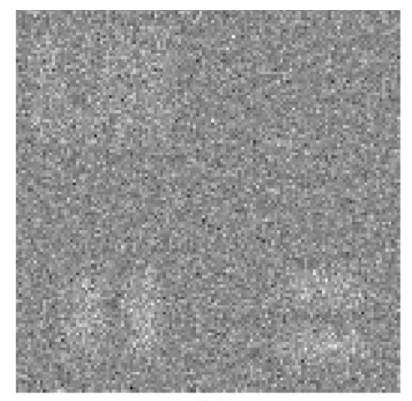

(b)

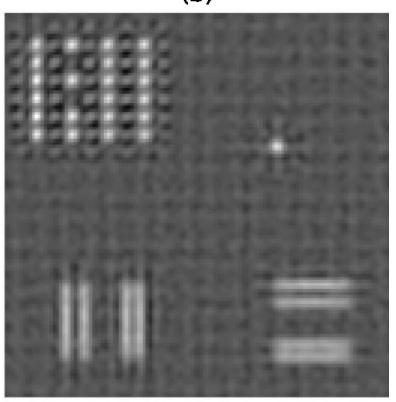

(d)
Fig. 4. High-resolution reconstructions from the images shown in Fig. 3 and with other directions of the patterned illumination. (a) Convolution of the object [Fig. 3(a)] with the widefield PSF and application of photon noise corresponding to $10^{4}$ expected photons at the maximum. (b) Application of a high-frequency enhancement ( $\gamma=2 \%)$, Eq. (10), to the image shown in (a). A simulation with a maximum of $10^{6}$ photons yielded a much clearer image (data not shown). (c) Reconstruction from simulated images with use of four directions of patterned illumination at nonsaturating excitation intensities, accounting for $m= \pm 1$ orders in the reconstruction. High-frequency enhancement was applied. (d) Reconstruction of SPEM data $[\alpha=5 / 6$, Eq. (11)] accounting for $m= \pm 3$ orders with a successive application of high-frequency enhancement ( $\gamma=2 \%$ ), Eq. (10). 


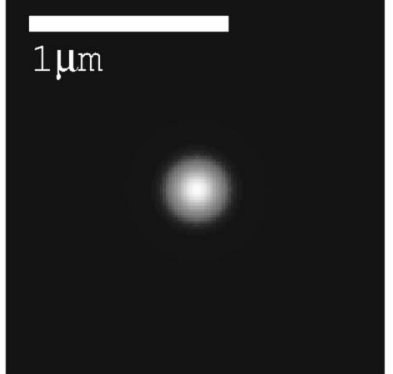

(a)

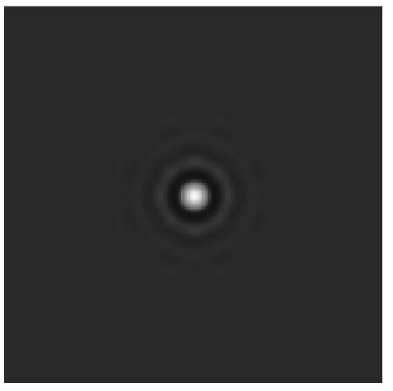

(c)

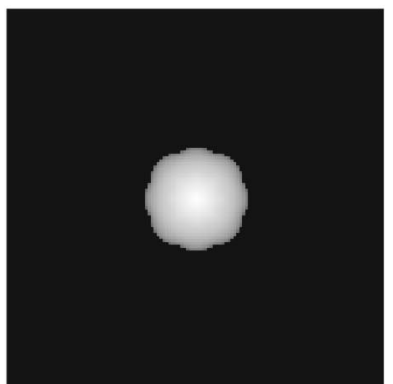

(e)

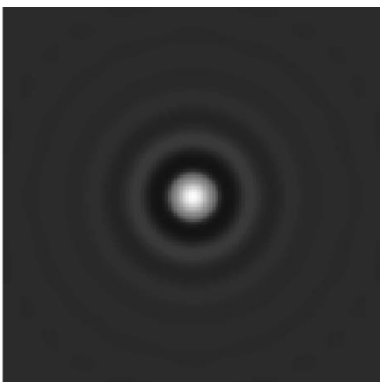

(b)

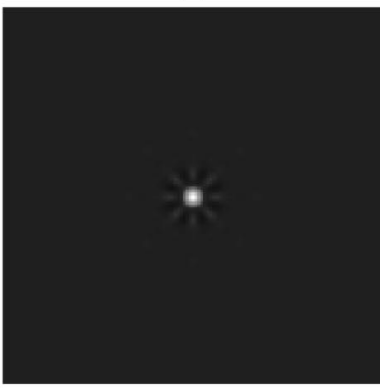

(d)

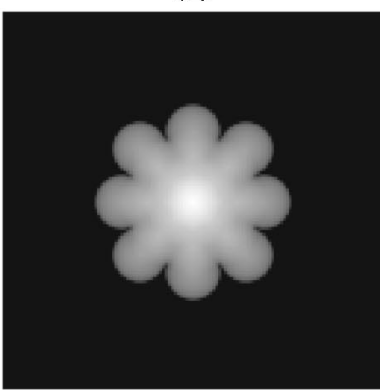

(f)
Fig. 5. Effective PSFs corresponding to different simulations. (a) Widefield PSF without filtering. (b) The result after filtering. (c) PSF including the reconstruction process in the linear case with four patterning directions and $m= \pm 1$ orders including high-frequency enhancement. (d) PSF with use of SPEM with four patterning directions and $m= \pm 3$ orders and successive filtering. (e), (f) Respective OTFs of (c) and (d).

tions would serve to close the gaps at high frequencies. Gaussians fitted to the PSF data (Fig. 6) yielded FWHMs of $215 \mathrm{~nm}$ for the unprocessed widefield PSF and $141 \mathrm{~nm}$ for widefield imaging with the high-frequency enhancement. The PSFs of the linear patterned excitation method yielded FWHM values of $142 \mathrm{~nm}$ (no filtering) and $83 \mathrm{~nm}$ (filtered at $\gamma=2 \%$ ). SPEM at the described saturation level gave FWHMs of $157 \mathrm{~nm}$ (unfiltered) and 61 $\mathrm{nm}$ (filtered at $\gamma=2 \%$ ). The SPEM PSF (without filtering) could not be fitted well with a Gaussian, thus yielding a slightly too large FWHM.

To emphasize the relevance of SPEM in potential biological applications, an inverted electron micrograph was used as input for the SPEM simulation (Fig. 7). As in Fig. 4, the input to the simulation [Fig. 7(a)] is compared with results of high-frequency-enhanced widefield microscopy [Fig. 7(b)], linear patterned excitation microscopy [Fig. 7(c)], and SPEM [Fig. 7(d)]. A slight nonuniformity in regions of the object of uniform intensity is observed (as in Fig. 4), which can probably be attributed to the nonisotropic OTF and the gaps at high frequency. A great improvement in resolution is observed in comparison with the linear methods.

\section{DISCUSSION}

By systematic saturation of the fluorophore(s) in SPEM a nonlinear relationship between the illumination intensity and the excitation probability of the fluorophore is established at every point in object space. This nonlinearity leads to the generation of higher spatial harmonics in the pattern of emittability. That is, components in Fourier space beyond the frequency limit defined by the Abbé condition are created. Because of the inherent properties of the microscope, the imaging process itself remains bounded in Fourier space, but the range of detectable spatial frequencies representing the object is extended (shifted) owing to the convolution of the object and emittability Fourier transforms.

A specific concern in SPEM, as in all fluorescence microscopy, is the potential influence of light-induced destruction of the fluorophore (photobleaching). Photobleaching generally originates from the two excited states, singlet and triplet. The latter is relatively long lived, even in oxygen-saturated water, and thus can react readily with oxygen. ${ }^{36}$ In SPEM, the light dose required to generate an image need not be greater than that for conventional microscopy. Thus the expected degree of photobleaching in SPEM and other forms of microscopy operated in the linear regime should be comparable. However, in SPEM, one may wish to apply the excitation in the form of a train of pulses of nanoseconds duration but with high intensity. In the event that excited-state absorption intervenes and contributes significantly to the overall photobleaching mechanism, an increased degree of photobleaching might be generated, although it might be possible to circumvent this effect by saturating the triplet instead of the singlet state. A further possible phenomenon is photodisruption due to high local absorption and inadequate thermal dissipation. However, one anticipates that the threshold for this physical process will be high, particularly if the triplet state is driven to saturation instead of (or in addition to) the singlet state.

A possible setup for imaging according to the SPEM principle and using a widefield microscope with epifluorescence illumination is depicted in Fig. 2(a). The patterned illumination is achieved by imaging an SLM [Fig. 2(a)] into the object plane via the tube lens and objective. The SLM can be a simple diffractive grating ${ }^{1,2,4}$ or a programmable device. To achieve a varying phase of the illumination pattern, the SLM can be translated (or programmed; see below) in small steps with respect to the nonmoving object. The minimum number of images required for a single reconstruction is determined by the number of unknown quantities in the associated linear system of equations. Changing the position of the pattern relative to the object can also be achieved by translating the object, which must be compensated for in the data reconstruction. Appropriate optical elements that alter the phase or amplitude of the light in the individual diffraction peaks can also be used to achieve the phase shifts. These can be placed near the back focal plane of the objective or at conjugate positions. Appropriate ap- 


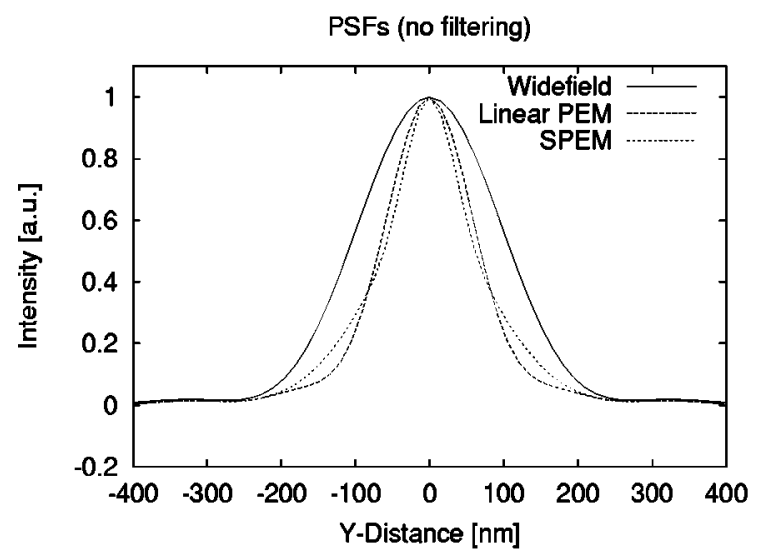

(a)

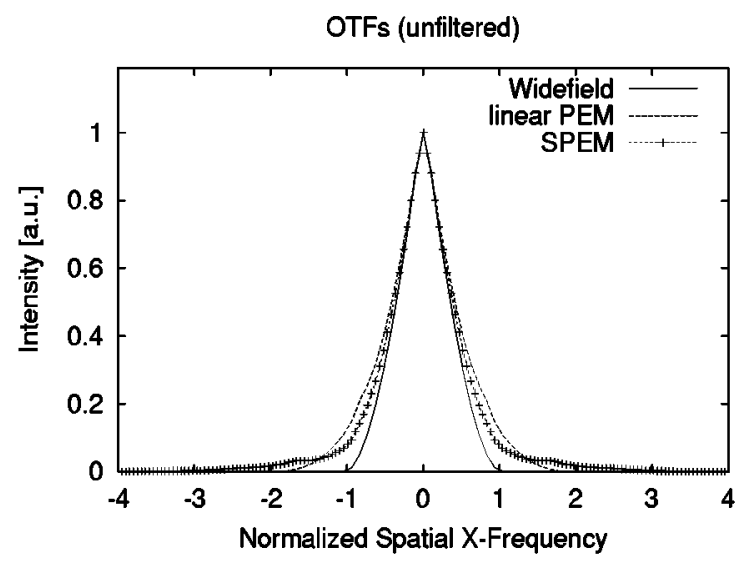

(c)

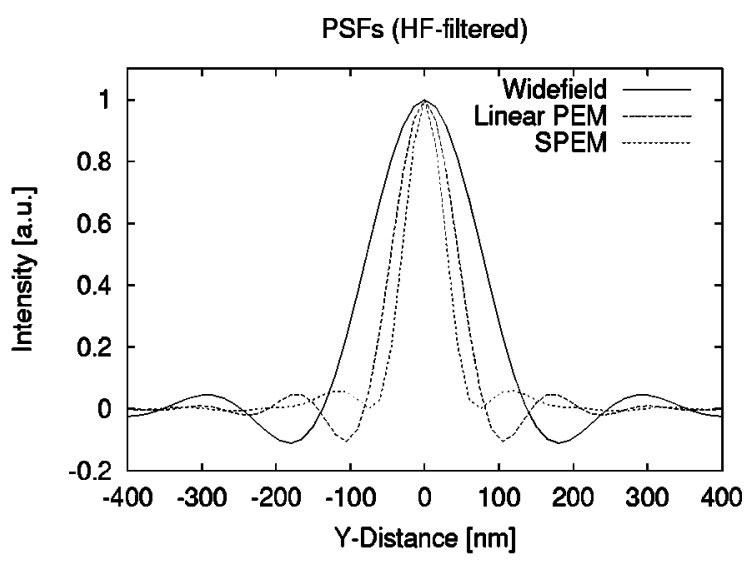

(b)

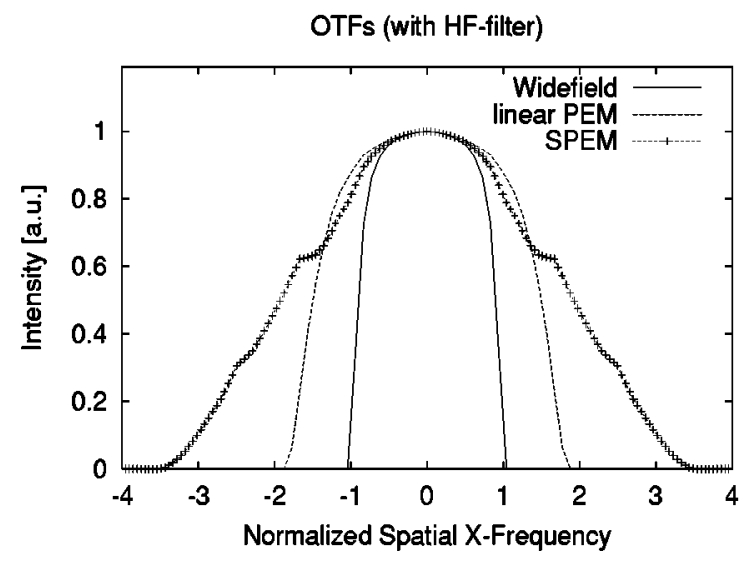

(d)

Fig. 6. One-dimensional cuts through the respective simulated (a), (b) PSFs or (c), (d) OTFs. (b), (d) With high-frequency enhancement $(\gamma=2 \%)$ and (a), (c) without high-frequency enhancement.

erture filters at these positions would serve to enhance contrast and constrain the patterns to the desired orders by selective suppression or attenuation of diffraction orders. For example, suppressing the 0 diffraction order in the case of a two-dimensional (2D) pattern yields a high contrast in the excitation pattern without removing the sectioning capabilities of the system [as it would in the case of a sinusoidal one-dimensional (1D) grating of maximum spatial frequency].

The simulated excitation method using a line grating and two (of its) diffraction orders for excitation is only one of many potential strategies for implementing SPEM. A programmable SLM will probably be very useful in the application, since it obviates the need for spatial movement and rotation of the excitation grating. We have described elsewhere ${ }^{37,38}$ a Programmable Array Microscope based on a digital micro-mirror device used simultaneously for illumination and detection. A particularly suitable device is a programmable phase-grating (or phase-mirror) primary directing the coherent light into the first two-dimensional diffraction orders of the programmed pattern. A series of images taken at varying excitation phases, (e.g., $\geqslant 25$ images for the reconstruction of a total of $5 \times 5$ orders) would suffice for reconstructing the object slice at high resolution. A setup such as that shown in Fig. 2(c) using a phase-modulating mirror would be expected to achieve a high light efficiency on the illu- mination side as well as maximum modulation depth. Other devices based on simultaneous illumination with multiple beam interference [Fig. 2(b)] also along the optic axis might be useful. Suitable light-sources could be a Q-switched Nd:YAG laser with an optional optical parametric oscillator for wavelength tunability or even flashlamps pumping the fluorophores into the triplet state.

Additional information about the sample, including reconstruction of three-dimensional (3D) images, can be gained by acquisition of a focus series. If the undiffracted 0 order is still present in the illumination light, the distribution of the latter inside the sample will also vary along the optic axis, leading to the corresponding $3 \mathrm{D}$ excitation structure in Fourier space. ${ }^{5,7}$ In analogy to the $1 \mathrm{D}$ and $2 \mathrm{D}$ cases, there will be peaks in the 3D Fourier transform to which the Fourier-transformed object will be attached. As in the $2 \mathrm{D}$ case, altering phases (mechanically or optically) in combination with the nonlinear effect would improve the axial resolution beyond the confocal Abbé diffraction limit.

The proposed method can also be combined with existing microscopy methods such as $I^{5}$ microscopy $^{17}$ or standing-wave microscopy. ${ }^{11,12}$ A possible setup could be that of Fig. 2(b). Laser light could in principle be used directly to form interferometer-like arrangements. In practice, a configuration with only a small spatial differ- 
ence between interfering beams might be advantageous. Illuminating the sample from the side [Fig. 2(b)] could be difficult but would provide the advantage of very high lateral spatial frequencies in the illumination pattern. Additional beams from below and above would also enhance axial spatial frequencies in the illumination pattern. Achieving the nonlinearity (fluorescence saturation) at high laser illumination intensities would permit reconstruction of 3D objects with previously unmatched resolution.

Whereas nonlinearities can enhance the resolution substantially, methods such as multiphoton microscopy yield at best only a limited improvement in resolution, since the primary illumination wavelength needs to be increased. More promising are other methods such as STED. Use of the saturation of the stimulated emission signal has led to a practical resolution improvement of $>3$ without the need for further image processing. ${ }^{30}$ However, the necessary optical setup is complicated. Femtosecond lasers are required, and the stimulated emission and excitation beam have to be well aligned. In addition, few dyes are suitable for excitation as well as for efficient stimulated emission while not being pumped to higher excitation levels. Other means for generating nonlinearities useful in resolution enhancement are fluorescence resonance energy transfer ${ }^{31}$ or fluorescence lifetime prolongation by repetitive excitation. ${ }^{32}$ These approaches do not require image processing for recovering the information but directly narrow the FWHM of the detected signal. Unfortunately, the necessary instruments are also complex.

A relevant question is what SNR is necessary to yield meaningful images by SPEM. As has been demonstrated in the simulations presented here, the method permits high-resolution reconstructions even at a moderate noise level corresponding to $\sim 10^{4}$ expected photons in the maximum of an image. In a way, SPEM can be viewed in real space as a technique of imaging with the negative spikes of the emittability pattern [Fig. 1(c)]. An alternative way of treating the data would then be to simply subtract a suitably scaled SPEM image from a widefield image (having constant emittability). The difference images could then be processed with techniques described by Benedetti et al., ${ }^{39}$ based on nonlinear approaches such as maximum projection. Alternatively, "virtual pinholes" at the positions of the emittability minima in the original SPEM image could be used. The latter approach would correspond closely to STED, except that the suppression of the unwanted fluorescence would be achieved computationally by subtraction of the SPEM emittability pattern. Such a subtractive approach would most probably be disadvantageous in terms of SNR in comparison with a properly performed STED experiment. However, this subtraction approach and the SPEM reconstruction introduced in this paper differ in one important respect. The decomposition into linear components with a succesive repositioning in Fourier space does not necessarily require a narrow FWHM in the emittability pattern but rather relies on the presence of high orders that contribute sufficiently to the detected signal, which corresponds to steep edges in the emittability pattern. With thick samples, the SPEM technique with use of a sinusoidal il- lumination pattern of high spatial frequency might be problematic, but in this case a pattern corresponding to the illumination with more sparsely distributed pinholes could be chosen. However, the acquisition of a greater number of images for decomposition into the many Fourier components would then be required.

The SPEM method has the further advantage of requiring only minor modifications to existing systems and the use of comparatively cheap pulsed lasers such as the Nd:YAG or even flashlamps. Since stimulated emission is not a feature of SPEM, every dye used in standard fluorescence microscopy should be suitable in principle, as long as it can be saturated at the excitation wavelength. In terms of SNR, methods using patterned illumination could well outperform confocal spot scanning methods that reject light at the pinhole. In SPEM the highspatial-frequency information can be detected more efficiently than with confocal methods, since the Fouriertransformed object is shifted in Fourier space into a region in which the OTF transmits more strongly. A further advantage is the use of a CCD camera as the detector, with a substantially higher quantum efficiency than that of a photomultiplier tube. In comparison with scanning methods, a CCD also provides a high degree of parallelization that increases the rate of image acquisition.

It is possible and advantageous to substitute the matrix technique (Appendix A) by algebraic iterative reconstruction methods operating directly on the raw data (such as maximum likelihood/expectation maximization). Additional orientations of the sinusoidal excitation distri-

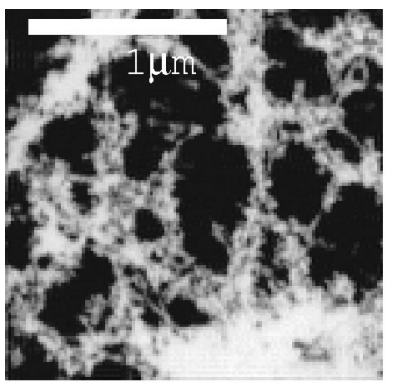

(a)

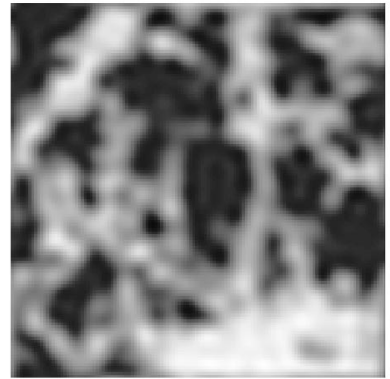

(c)

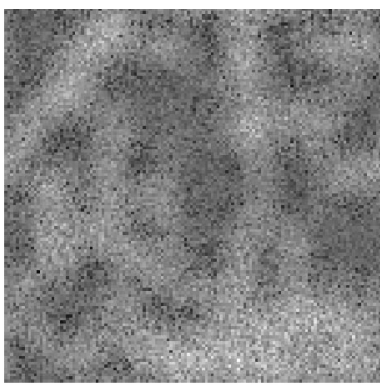

(b)

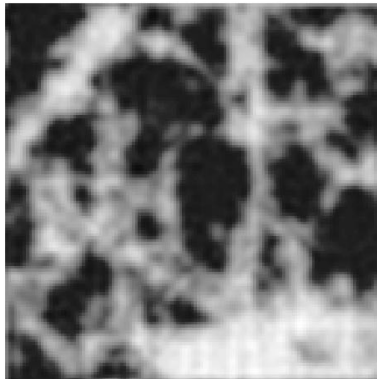

(d)
Fig. 7. Simulated application of the SPEM concept to a slice of a cell nucleus. (a) Intensity-inverted part of an electron micrograph of an embryonal bovine cell nucleus near its nuclear membrane displaying the "nuclear matrix." Image (a) has been used for SPEM simulations in which every image was adjusted to a maximum of $10^{4}$ expected photons. (b) Simulated fluorescence widefield image with successive high-frequency filtering $(\gamma$ $=2 \%$ ). (c) Reconstruction using linear patterned excitation microscopy ( $10^{4}$ photons in maximum, $m= \pm 1$ orders) including high-frequency enhancement $(\gamma=2 \%)$. (d) SPEM reconstruction with high-frequency enhancement $(\gamma=2 \%)$. 
bution and a Fourier-filtering approach (high-frequency enhancement) modified for application in two dimensions should eliminate the problem of residual patterning in the resultant images and further enhance the resolution.

The SPEM strategy can also be applied to various other methods that exhibit nonlinearity. For example, at ultrashort time scales, Rabi oscillations ${ }^{40}$ that modulate the sample fluorescence may be possible. In material sciences, i.e., in investigation of gases, semiconductors, or cooled particle traps, this concept of resolution improvement might prove very useful, although the required conditions are probably hard to achieve in a biologically relevant context owing to the usually short dephasing times.

Other nonlinear effects can be used for resolution improvement if their Taylor series expansion [relation (5)] contains terms of high-enough orders. Even in the case of multiple linear dependences of the emission intensity on spatially dependent factors [e.g., $I_{\mathrm{ex}}(\mathbf{x})$ and an independent influence $b(\mathbf{x})$, such as a static electromagnetic field], mixed terms such as $c_{4 b} I_{\text {ex }}(\mathbf{x}) b(\mathbf{x})$ arise, leading to synergetic effects on the improvement in resolution. Other possibilities are stimulated emission at a different frequency, the Raman effect, or pressure modulation. In the event that the imaging process includes a coherent component, the theoretical treatment has to be adapted accordingly.

\section{APPENDIX A: IMAGE RECONSTRUCTION FROM THE ACQUIRED DATA}

After acquiring the data containing information about high spatial frequencies of the object, processing is required to yield a high-resolution image. To do this, it is necessary to distinguish between the emittability pattern $\operatorname{Em}(\mathbf{x})$ and the density distribution describing the object, $\rho(\mathbf{x})$ [relation (6)]. This can be achieved by varying the $\operatorname{Em}(\mathbf{x})$ at each spatial position $\mathbf{x}$. If $I_{\mathrm{ex}}(\mathbf{x})$ is a spatial raster, it can be shifted stepwise with respect to the object, taking an image at every raster position. Another approach is to alter the strength or the shape of the spatial distribution in $I_{\mathrm{ex}}(\mathbf{x})$ between individual images. Both methods permit the separation of the individual components given in the Taylor expansion [relation (5)]. If one illuminates with a periodic pattern, the emitted light can be reformulated as the sum of object components attached to single $\delta$ peaks [Figs. 1(b) and 1(d)]. Thus the recorded image is the sum over all imaged components,

$$
\widetilde{h_{\mathrm{em}}}(\mathbf{k})\left[\phi_{l} \delta\left(\mathbf{k}-\mathbf{\Delta} \mathbf{k}_{l}\right) \otimes \widetilde{\rho}(\mathbf{k})\right],
$$

of which only one is shown in the figure. $\Delta \mathbf{k}_{l}$ marks the position of the object's zero frequency of this shifted component in Fourier space. The complex-valued coefficients $\phi_{l}$ are determined by the position and shape of $I_{\mathrm{ex}}(\mathbf{x})$ and by the coefficients $c_{n}$ in relation (6). The complex-valued coefficients account for the phases and strength of the $\delta$ peaks.

Multiplication with the detection OTF does not prevent the definition and solution of this system of equations at every point in Fourier space within the region of support of the detection OTF. By shifting the individual determined components back in Fourier space by $-\mathbf{\Delta} \mathbf{k}_{l}$ [or multiplying with $\exp \left(-i \Delta \mathbf{k}_{l} \cdot \mathbf{x}\right)$ in real space] and adding them with individual weights (possibly dependent on $\mathbf{k}$ ), one can obtain a high resolution data set. This can further be processed with linear or nonlinear filters or by deconvolution techniques to suppress artifacts and improve resolution further.

A spatial sinusoidal distribution of the excitation intensity pattern (including a constant offset to yield only positive intensity values) in the linear case [Fig. 1(a)] leads to three distinct ( $\delta$-distribution-like) maxima in its Fourier transform $\left[\mathbf{k}_{0}=0, \mathbf{k}_{+1}=+\mathbf{k}_{b}\right.$ and $\mathbf{k}_{-1}=-\mathbf{k}_{b}$ in Fig. 1(b)]. Depending on the modulation depth and the actual phase of the illumination pattern, these maxima have a well-defined amplitude and phase in the complex plane. Owing to the nonlinear dependence of the emission intensity on the illumination intensity caused by fluorescence saturation, a distinct pattern of emittability $\operatorname{Em}(\mathbf{x})$ is obtained for a specific fluorophore. In Fourier space this pattern contains an infinite series of maxima, the complex magnitudes of which, however, decrease rapidly for higher $\mathbf{k}$ values [Fig. 1(d)]. For the reconstructions presented here, a 1D grating structure was assumed for illumination and only a finite number of maxima $\mathbf{k}_{ \pm \max }$ $= \pm m \mathbf{k}_{b}$ were accounted for, neglecting higher orders. If the illumination intensity distribution, and thus the emittability pattern, is translated in space, the complex phase of the individual peaks in Fourier space change accordingly. Accounting for $\pm m$ maxima (and the one at $\mathbf{k}_{0}$ $=0), \geqslant(s=2 m+1)$ images taken under different conditions are necessary to separate the individual components of the Fourier-transformed object belonging to the convolution with an individual maximum (i.e., shifted in Fourier space). In the simulated example, the number of maxima considered in the reconstruction was $m=3$. The complex phase angles of such maxima in Fourier space are proportional to $l \mathbf{k}_{b} \cdot \Delta \mathbf{x}, l$ indexing the maxima, when the illumination intensity distribution is shifted by $\Delta \mathbf{x}$, since a shift by $\Delta \mathbf{x}$ is equivalent to a multiplication by $\exp (i \mathbf{k} \cdot \Delta \mathbf{x})$ in Fourier space. If $s$ (in our simulations $s=7$ ) different microscope images $I_{n}(\mathbf{x})$, with their Fourier transforms $\widetilde{I_{n}}(\mathbf{k})$, are obtained by shifting the phase of the illumination (and thus the emittability) distribution by steps of $1 / s$, the following system of equations is obtained for every $\mathbf{k}$ position in Fourier space:

$$
\begin{gathered}
\widetilde{I_{n}}(\mathbf{k})=\sum_{l=-m}^{m} M_{\ln \widetilde{\rho}_{l}(\mathbf{k}),} \\
M_{\ln }=c_{l}^{\prime} \exp (2 \pi i \ln / s), \\
l \in\{-m \ldots m\}, \quad n \in\{0 \ldots s-1\} .
\end{gathered}
$$

The $\widetilde{I_{n}}(\mathbf{k})$ represent the Fourier-transformed microscopic images measured for a specific spatial position (phase) of the emittability pattern; $\widetilde{\rho}_{l}(\mathbf{k})$ denotes the Fourier-transformed complex-valued object components belonging to the $l$ th maximum of the emittability pattern, after transmission and alteration by the OTF of the imaging system. These transmitted object components remain shifted in Fourier space by $\Delta \mathbf{k}_{l}=l \mathbf{k}_{b}$ relative to the original object $\widetilde{\rho}(\mathbf{k})$. Solving this system of equations, 
e.g., by inverting the matrix $M$, leads to the individual transmitted object components $\widetilde{\rho}_{l}(\mathbf{k})$.

The real-valued coefficients $c_{l}^{\prime}$ [Eqs. (A1)-(A3)] are selected in a manner that ensures a nonsingular matrix, i.e., by acquiring a suitable number of recorded images. When the $c_{l}^{\prime}$ are exceedingly small, the associated extracted Fourier component is dominated by noise, leading to difficulties in the interpretation of the reconstructed images. Thus, very small $c_{l}^{\prime}$ are omitted in the reconstruction. At the other extreme, if a given nonlinearity generates very high values of $c_{l}^{\prime}$, which cannot be accounted for in the reconstruction, problems may arise. Other means of data reconstruction, such as maximumlikelihood-based techniques, may be better suited for dealing with such a situation.

Owing to the linearity of the Fourier transformation, the calculation can also be performed pixel by pixel in real space. Each complex-valued component $\widetilde{\rho}_{l}(\mathbf{k})$ is then shifted in Fourier space by the vector $-\Delta \mathbf{k}_{l}$ such that individual $\widetilde{\rho}_{l}(\mathbf{k})$ end up in the position where they can be measured by using a constant flat illumination. This Fourier-space shift can be performed in real space by a multiplication of $\rho_{l}(\mathbf{x})$ by $\exp \left(-i \Delta \mathbf{k}_{l} \cdot \mathbf{x}\right)$, thereby completely avoiding Fourier transformations in the reconstruction process. Equation (A3) does not account for the absolute position $\left(\Delta \mathbf{x}_{0}\right)$ of the excitation pattern [defined by the symmetry axis of the first pattern $\left.I_{0}(\mathbf{x})\right]$ with respect to the real-space coordinate system. $\Delta \mathbf{x}_{0}$ either is known a priori or can be extracted from the data, after which the components $\widetilde{\rho}_{l}(\mathbf{k})$ are corrected by multiplication by $\exp \left(-i l \mathbf{k}_{b} \cdot \Delta \mathbf{x}_{0}\right)$. These reconstructed components $\widetilde{\rho_{l}^{\text {rec }}}(\mathbf{k})$ are added by using weights to finally yield a high-resolution image of the sample. The weights may be altered from pixel to pixel in Fourier space to optimize for SNR and to compensate for possible artifacts in the experiment and "apodize" the OTF of the total system. A final OTF defining this apodization can be chosen in the $2 \mathrm{D}$ case, for example, as $\widetilde{h_{\text {filtered }}}=\sin \left(\pi|k|+\left|k_{\max }\right|\right.$ $\left.2\left|k_{\max }\right|\right)$ with the maximum reconstructed spatial frequency $k_{\max }$ to minimize the second moment of the PSF projected along any in-plane direction. ${ }^{41,42}$

The method for reconstruction described above can be applied to data acquired (2D or $3 \mathrm{D}$ ) with a $1 \mathrm{D}$ structure for illumination, successively pointed in different directions. Since the zero-order object component remains unchanged, $2 m$ images are sufficient for each reconstruction of illumination directions succeeding the first. Two(or three-) dimensional emittability patterns can, however, also be generated and shifted in different directions of space, yielding a correspondingly larger system of equations to retrieve the multiple orders in two (or three) dimensions.

\section{ACKNOWLEDGMENTS}

The authors thank S. W. Hell for useful discussions. R. Heintzmann was the recipient of a postdoctoral fellowship of the Max Planck Society. Many thanks go to A. Egner for supplying the program for the computation of the widefield PSFs using vector theory. Jéril Degruard provided the electron micrograph that was used in the simulation (Fig. 7).
Corresponding author R. Heintzmann's e-mail address is rheintz@gwdg.de.

\section{REFERENCES AND NOTES}

1. R. Heintzmann and C. Cremer, "Lateral modulated excitation microscopy: improvement of resolution by using a diffraction grating," in Optical Biopsies and Microscopic Techniques III, I. J. Bigio, H. Schneckenburger, J. Slavik, K. Svanberg, and P. M. Viallet, eds., Proc. SPIE 3568, 185-196 (1999).

2. M. G. L. Gustafsson, "Surpassing the lateral resolution limit by a factor of two using structured illumination microscopy,” J. Microsc. 198, 82-87 (2000).

3. T. Wilson, R. Juskaitis, and M. A. A. Neil, "A new approach to three dimensional imaging in microscopy," Cell Vision $\mathbf{4}$, 231 (1997)

4. M. A. A. Neil, R. Juskaitis, and T. Wilson, "Method of obtaining optical sectioning by using structured light in a conventional microscope," Opt. Lett. 22, 1905-1907 (1997).

5. M. G. L. Gustafsson, "Doubling the lateral resolution of wide-field fluorescence microscopy using structured illumination," in Three-Dimensional and Multidimensional Microscopy: Image Acquisition Processing VII, J. Conchello, C. J. Cogswell, and T. Wilson, eds., Proc. SPIE 3919, 141150 (2000).

6. J. T. Frohn, H. F. Knapp, and A. Stemmer, "True optical resolution beyond the Rayleigh limit achieved by standing wave illumination," Proc. Natl. Acad. Sci. USA 97, 72327236 (2000)

7. J. T. Frohn, H. F. Knapp, and A. Stemmer, "Threedimensional resolution enhancement in fluorescence microscopy by harmonic excitation," Opt. Lett. 26, 828-830 (2001).

8. M. Nagorni and S. W. Hell, "Coherent use of opposing lenses for axial resolution increase in fluorescence microscopy. I. comparative study of concepts," J. Opt. Soc. Am. A 18, 36-48 (2001).

9. M. Minsky, "Microscopy apparatus," U.S. patent 3,013,467, December 19, 1961.

10. F. Lanni, B. Bailey, D. L. Farkas, and D. L. Taylor, "Excitation field synthesis as a means for obtaining enhanced axial resolution in fluorescence microscopes," Bioimaging 1, 187196 (1993)

11. F. Lanni, D. L. Taylor, and B. Bailey, "Field synthesis and optical subsectioning for standing wave microscopy," U.S. patent 5,801,881, September 1, 1998.

12. B. Albrecht, A. V. Failla, R. Heintzmann, and C. Cremer "Spatially modulated illumination microscopy: online visualization of intensity distribution and prediction of nanometer precision of axial distance measurements by computer simulations," J. Biomed. Opt. 6, 292-299 (2001).

13. S. W. Hell, S. Lindek, C. Cremer, and E. H. K. Stelzer, "Measurement of the 4pi-confocal point spread function proves $75 \mathrm{~nm}$ axial resolution," Appl. Phys. Lett. 64, 13351337 (1994).

14. S. W. Hell, M. Schrader, and H. T. M. van der Voort, "Farfield fluorescence microscopy with three-dimensional resolution in the $100 \mathrm{~nm}$ range," J. Microsc. 185, 1-5 (1997)

15. M. G. L. Gustafsson, D. A. Agard, and J. W. Sedat, " $3 \mathrm{D}$ widefield microscopy with two objective lenses: experimental verification of improved axial resolution," in ThreeDimensional Microscopy: Image Acquisition and Processing III, C. J. Cogswell, G. S. Kino, and T. Wilson, eds., Proc. SPIE 2655, 62-66 (1996).

16. M. Gustafsson, J. Sedat, and D. Agard, "Method and apparatus for three-dimensional microscopy with enhanced depth resolution," U.S. patent 5,671,085, September 23, 1997.

17. M. G. L. Gustafsson, D. A. Agard, and J. W. Sedat, "I ${ }^{5}$ M: 3D widefield light microscopy with better than $100 \mathrm{~nm}$ axial resolution,” J. Microsc. 195, 10-16 (1999).

18. R. Juskaitis, T. Wilson, M. A. A. Neil, and M. Kozubek, "Ef- 
ficient real-time confocal microscopy with white licht sources," Nature (London) 383, 804-806 (1996).

19. T. Wilson, R. Juskaitis, M. A. A. Neil, and M. Kozubeck, "An aperture correlation approach to confocal microscopy," in Three-Dimensional Microscopy: Image Acquisition and Processing IV, C. J. Cogswell, J. Conchello, and T. Wilson, eds., Proc. SPIE 2984, 21-23 (1997).

20. In the literature patterned excitation techniques have also been named structured illumination microscopy, harmonic excitation light microscopy (HELM) and laterally modulated excitation (LMEM).

21. P. J. Shaw, D. A. Agard, Y. Hirakoa, and J. W. Sedat, "Tilted view reconstruction in optical microscopy: three dimensional reconstruction of drosophila melanogaster embryo nuclei," Biophys. J. 55, 101-110 (1989).

22. R. Heintzmann, G. Kreth, and C. Cremer, "Reconstruction of axial tomographic high resolution data from confocal fluorescence microscopy-a method for improving 3D FISH images," Anal. Cell Pathol. 20, 7-15 (2000).

23. R. Heintzmann and C. Cremer, "Axial tomographic confocal fluorescence microscopy” J. Microsc. 206, 7-23 (2002).

24. K. Ichie, "Laser scanning optical system and laser scanning optical apparatus," U.S. patent 5,796,112, August 18, 1998.

25. P. Hänninen and S. Hell, "Luminescence-scanning microscopy process and a luminescence scanning microscope utilizing picosecond or greater pulse lasers," U.S. patent $5,777,732$, July $7,1998$.

26. W. Denk, J. H. Strickler, and W. W. Webb, "Two-photon fluorescence scanning microscopy," Science 248, 73-76 (1990).

27. S. W. Hell and M. Kroug, "Ground-state depletion fluorescence microscopy, a concept for breaking the diffraction resolution limit," Appl. Phys. B 60, 495-497 (1995).

28. S. W. Hell, "Increasing the resolution of far-field fluorescence light microscopy by point-spread-function engineering," in Topics in Fluorescence Spectroscopy: Nonlinear and Two-Photon-Induced Fluorescence, J. Lakowicz, ed. (Plenum, New York, 1997), Vol. 5, pp. 361-426.

29. S. W. Hell and J. Wichmann, "Breaking the diffraction resolution limit by stimulated emission: stimulated-emissiondepletion fluorescence microscopy," Opt. Lett. 19, 780-782 (1994).

30. T. A. Klar, S. Jakops, M. Dyba, and S. W. Hell, "Fluorescence microscopy with diffraction resolution barrier broken by stimulated emission," Proc. Natl. Acad. Sci. USA 97, 8206-8210 (2000).

31. A. Schönle, P. E. Hänninen, and S. W. Hell, "Nonlinear fluorescence through intermolecular energy transfer and resolution increase in fluorescence microscopy," Ann. Phys. (Leipzig) 8, 115-133 (1999).

32. A. Schönle and S. W. Hell, "Far-field fluorescence microscopy with repetitive excitation," Eur. Phys. J. D 6, 283-290 (1999).

33. D. R. Sandison, R. M. Williams, K. S. Wells, J. Strickler, and W. W. Webb, "Quantitative fluorescence confocal laser scanning microscopy (CLSM)," in Handbook of Biological Confocal Microscopy, 2nd ed., J. B. Pawley, ed. (Plenum, New York, 1995), pp. 47-50.

34. R. Y. Tsien and A. Waggoner, "Fluorophores for confocal microscopy," in Handbook of Biological Confocal Microscopy, 2nd ed., J. B. Pawley, ed. (Plenum, New York, 1995), pp. $267-268$.

35. A. Egner and S. W. Hell, "Equivalence of the HuygensFresnel and Debye approach for the calculation of high aperture point-spread functions in the presence of refractive index mismatch," J. Microsc. 193, 244-249 (1999).

36. R. Y. Tsien and A. Waggoner, "Fluorophores for confocal microscopy," in Handbook of Biological Confocal Microscopy, 2nd ed., J. B. Pawley, ed. (Plenum, New York, 1995), pp. 269, 272.

37. Q. S. Hanley, P. J. Verveer, M. J. Gemkov, D. Arndt-Jovin, and T. M. Jovin, "An optical sectioning programmable array microscope implemented with a digital micromirror device," J. Microsc. 196, 317-331 (1999).

38. R. Heintzmann, Q. S. Hanley, D. Arndt-Jovin, and T. M. Jovin, "A dual path programmable array microscope (PAM): simultaneous acquisition of conjugate and non-conjugate images," J. Microsc. 204, 119-137 (2001).

39. P. A. Benedetti, V. Evangelista, D. Guidarini, and S. Vestri "Method for the acquisition of images by confocal," U.S. patent 6,016,367, January 18, 2000.

40. M. Sargent III, M. O. Scully and W. E., Jr., Lamb, Laser Physics (Addison-Wesley, London, 1982) (4th printing).

41. P. J. Sementilli, B. R. Hunt, and M. S. Nadar, "Analysis of the limit to superresolution in incoherent imaging," J. Opt. Soc. Am. A 10, 2265-2276 (1993).

42. D. Gabor, "Theory of communication," J. Inst. Electr. Eng. 63, 429-457 (1946). 